\title{
Characterization of infections and hypogammaglobulinemia treated with the combination of pertuzumab and trastuzumab
}

\author{
Joanne E. Mortimer ${ }^{1}$ (1) $\cdot$ Laura Kruper ${ }^{2} \cdot$ Jae Jung $^{3} \cdot$ Louise Wong $^{1} \cdot$ Jill Cooper $^{1} \cdot$ Daphne Stewart $^{1} \cdot$ Samuel Chung $^{1}$. \\ Kim Wai Yu ${ }^{4}$. Sanjeet Dadwal ${ }^{5} \cdot$ Yuan Yuan ${ }^{1}$
}

Received: 16 September 2019 / Accepted: 9 October 2019 / Published online: 22 October 2019

(c) The Author(s) 2019

\begin{abstract}
Purpose We update a patient series that reported a high incidence of infection with Gram-positive cocci in women treated with the combination of pertuzumab and trastuzumab and further characterize this clinical problem.

Patients Treating physicians and advanced practice partners identified women who developed infections while on treatment with pertuzumab and trastuzumab alone or in combination with chemotherapy and enrolled them onto this registry trial.

Results Between March, 2014 and May, 2017, 48 patients with HER2-positive breast cancers were reported to have 59 individual infections. The median age was 48 years. Twenty-four patients received neoadjuvant therapy, 17 were treated for metastatic disease, and 7 were treated in the adjuvant setting. Pertuzumab and trastuzumab were combined with carboplatin and docetaxel in $24(49 \%)$ patients, docetaxel in $10(21 \%)$, nab-paclitaxel in $12(24 \%)$, and without other agents in $2(4 \%)$. Granulocyte growth factors were administered in $24(49 \%)$ patients and no patients were documented to be neutropenic. Folliculitis developed in $25(52 \%)$ patients and was counted as a single infection. Abscesses developed at a number of sites in $24(49 \%)$ patients, including a septic knee requiring total knee replacement. Paronychia occurred in 7 (15\%) patients, and $5(10 \%)$ developed cellulitis. When cultures were obtained, Gram-positive cocci were consistently identified. Hypogammaglobulinemia was documented in 14 (36\%) of the 33 patients tested.

Conclusions Our data continue to support an increased risk of infections with Gram-positive cocci as a potentially serious adverse event in women treated with pertuzumab and trastuzumab.
\end{abstract}

Keywords Infections $\cdot$ Hypogammaglobulinemia $\cdot$ Pertuzumab $\cdot$ Trastuzumab

Joanne E. Mortimer

jmortimer@coh.org

1 Department of Medical Oncology and Experimental Therapeutics, City of Hope Comprehensive Cancer Center, 1500 East Duarte Rd, Duarte, CA 91010, USA

2 Department of Surgery, City of Hope Comprehensive Cancer Center, 1500 East Duarte Rd, Duarte, CA 91010, USA

3 Department of Dermatology, City of Hope Comprehensive Cancer Center, 1500 East Duarte Rd, Duarte, CA 91010, USA

4 Department of Pharmacy, City of Hope Comprehensive Cancer Center, 1500 East Duarte Rd, Duarte, CA 91010, USA

5 Department of Infection Disease, City of Hope Comprehensive Cancer Center, 1500 East Duarte Rd, Duarte, CA 91010, USA

\section{Introduction}

We previously described the development of unique infections involving the skin and nails that we attributed to the addition of pertuzumab to trastuzumab-based chemotherapy in women with HER2-positive breast cancer. This problem often developed in association with hypogammaglobulinemia [1]. This report updates and expands our initial series, providing longer follow-up and further characterization of the problem.

\section{Methods}

From March, 2014 until May, 2017, women who developed infections while on chemotherapy regimens that included both pertuzumab and trastuzumab were enrolled onto a registry trial approved by the Institutional Review Board 
(IRB). Patients were identified by the treating physician or advanced practice partner. Following our initial report, the care team had a heightened awareness of the potential for infectious complications and subsequent patients were generally identified and reported prospectively. The patients in this manuscript include the 18 women previously reported [1].

The clinical workup of the patients was not defined. Because we reported hypogammaglobulinemia in many of the initial patients, quantitative immunoglobulins were frequently checked at the time of the infection. Antimicrobial treatment was determined by the treating clinicians on a case-by-case basis, and the departments of infectious disease and/or dermatology were often consulted for treatment recommendations.

An episode of infection was defined as an infection that prompted attention to the treating oncologist or advanced practice partner. Folliculitis was the most common, frequently involved multiple sites and it often recurred with subsequent cycles of treatment. We counted folliculitis as a single infection, even if the patient developed additional eruptions with subsequent cycles of treatment. In instances where the attribution of the infection to systemic therapy was in question, the infectious disease consultant (SD) provided the attribution.

\section{Results}

\section{Characteristics and demographics}

Forty-eight patients developed 59 individual episodes of infection. Their characteristics are summarized in Table 1. The median age was 48 years. Twenty-four (50\%) patients received pertuzumab/trastuzumab-based chemotherapy in the neoadjuvant setting, 17 (35\%) were treated for metastatic disease, and 7 (15\%) were treated in the adjuvant setting. Two patients developed infections while on trastuzumab and pertuzumab alone, and 46 received treatment that included a taxane with 25 also receiving carboplatin. The majority of patients received granulocyte colony-stimulating factor (GCSF) agents. None of the patients were neutropenic when they presented with infection.

\section{Folliculitis}

The most common infection was folliculitis, which developed in $25(52 \%)$ patients within $2-7$ days of treatment. The areas of involvement included scalp (13), face (9; 2 also had in chest), abdomen (2), and arm (1). The skin eruptions were self-limited and frequently recurred with each additional cycle. Since our initial report, we have treated infections like EGFR drug reactions using topical clindamycin or
Table 1 Patient demographics

\begin{tabular}{|c|c|}
\hline Number of patients & 48 \\
\hline Age & 48 years $(25-73)$ \\
\hline \multicolumn{2}{|l|}{ Setting } \\
\hline Metastatic disease & 17 \\
\hline Neoadjuvant & 24 \\
\hline Adjuvant & 7 \\
\hline \multicolumn{2}{|l|}{ Regimen } \\
\hline PTH & 10 \\
\hline PTCH & 24 \\
\hline nabPT & 12 \\
\hline $\mathrm{PH}$ & 2 \\
\hline \multicolumn{2}{|c|}{ Type of infection (often multiple sites) } \\
\hline Abscess & 24 patients \\
\hline Breast & 1 \\
\hline Skin & 1 \\
\hline Axilla & 1 \\
\hline Port & 2 \\
\hline Dental & 1 \\
\hline Vaginal & 1 \\
\hline Breast wound/seroma & 2 \\
\hline Chest wall & 1 \\
\hline Thigh & 1 \\
\hline Thumb & 1 \\
\hline Buttocks & 4 \\
\hline Shoulder & 1 \\
\hline Septic arthritis & $1^{*}$ \\
\hline Paronychia & 7 \\
\hline Folliculitis & 25 patients \\
\hline Scalp & 13 \\
\hline Face & 9 \\
\hline Abdomen & 2 \\
\hline Arm & 1 \\
\hline Paronychia & 7 \\
\hline Cellulitis & 5 \\
\hline
\end{tabular}

PTCH pertuzumab, trastuzumab, carboplatin, and docetaxel, PTH pertuzumab, trastuzumab, and docetaxel, nabPT nab-paclitaxel, pertuzumab, and trastuzumab, $\mathrm{PH}$ pertuzumab and trastuzumab

*Patient required a total knee replacement

doxycycline vs oral or parenteral antibiotics for more severe infections. Examples of the folliculitis are shown in Fig. 1.

\section{Deep-seated infections}

Abscesses developed at different sites in 24 patients: buttocks (4) and one each involving the breast, skin, axilla, vagina, chest wall, thigh, thumb, and shoulder. Two patients developed postoperative wound infections requiring additional surgery. Two patients developed portacath infections within 2 days of catheter placement and required removal of the device. One patient developed a dental abscess and had 
Fig. 1 Folliculitis: a trunk and back; b scalp; c large pustule on arm
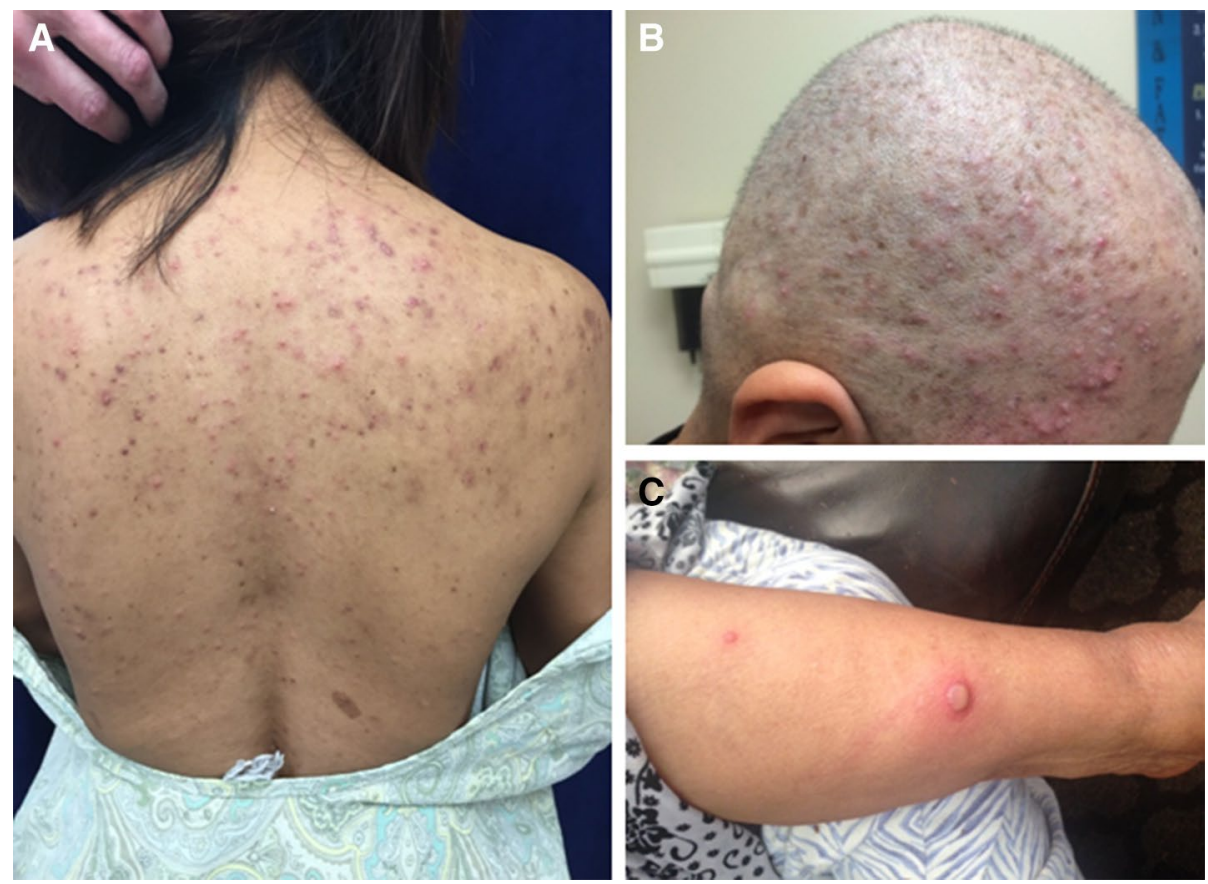

recurrent episodes of Streptococcus agalactiae bacteremia. Her case is discussed in detail below. Most abscesses were treated successfully with antibiotics. One of the patients who had an infected portacath developed tenderness in a prosthetic knee. She was initially treated conservatively with intravenous vancomycin but eventually required removal and replacement of the prosthesis. Cultures preoperatively were negative but methicillin-sensitive Staphylococcus aureus was cultured at surgery.

Severe paronychial infections were observed in 7 (15\%) patients and required surgical intervention in 1. Cellulitis was observed in $5(10 \%)$ patients and was treated with conservatively with antibiotics.

Most patients were treated for presumed Staphylococcus infections without obtaining cultures. Of the 21 infectious episodes for which cultures were obtained, all had infection with Gram-positive bacteria. Methicillin-sensitive Staphylococcus aureus (MSSA) was identified in seven patients, methicillin-resistant Staphylococcus aureus (MRSA) in eight, Streptococcus agalactiae in three (same patient), and Enterobacter faecalis in one (toe infection). The cultures were negative in two patients who had already been initiated on antibiotics.

\section{Fatality}

A 58-year-old woman with a locally advanced estrogen receptor positive $(E R+)$, progesterone receptor positive $(\mathrm{PR}+)$, HER2-positive cancer received her first cycle of pertuzumab, trastuzumab, carboplatin, and docetaxel (PTCH) without incidence. She was seen on day 1 of cycle 2 with a
WBC of 13.0 (11.7 ANC) and received Neulasta ${ }^{\circledR}$ on day2. On day 6 , her family reported a sudden change in mental status and she arrived at the local ED in full arrest and could not be resuscitated. No blood was drawn to assess the WBC or obtain cultures. At autopsy, the cause of death was ascribed to sepsis from Gram-positive cocci, which was not speciated.

\section{Hypogammaglobulinemia}

Because of the high incidence of Gram-positive cocci infections, documented or suspected, we questioned whether patients were hypogammaglobulinemic and began measuring quantitative immunoglobulins. Because this was a registry trial, there was no requirement for assessing immunoglobulins and they were measured at different time periods throughout chemotherapy. Of the 33 patients tested for immunoglobulin levels, 14 (36\%) had abnormally low levels; IgG was low in 6; IgM in 3, and IgA in 2. Both IgG and IgM were low in 1 and all three immunoglobulins were low in 1.

The following two patients illustrate the impact of pertuzumab on protein levels and infection. The course of a 52-year-old woman (Patient 1) who was treated for locally advanced breast cancer is summarized in Fig. 2, charting her total protein and immunoglobulin levels. On day 14 following cycle 1 of PTCH, she was emergently admitted to the hospital with a facial rash with pustules, loss of consciousness, hypotension, and decreased urine output. On admission, her WBC was 25.3 and she had a Scr of 16.63. She was treated with vasopressors and ceftriaxone with improvement in hemodynamics and renal function without the need for 


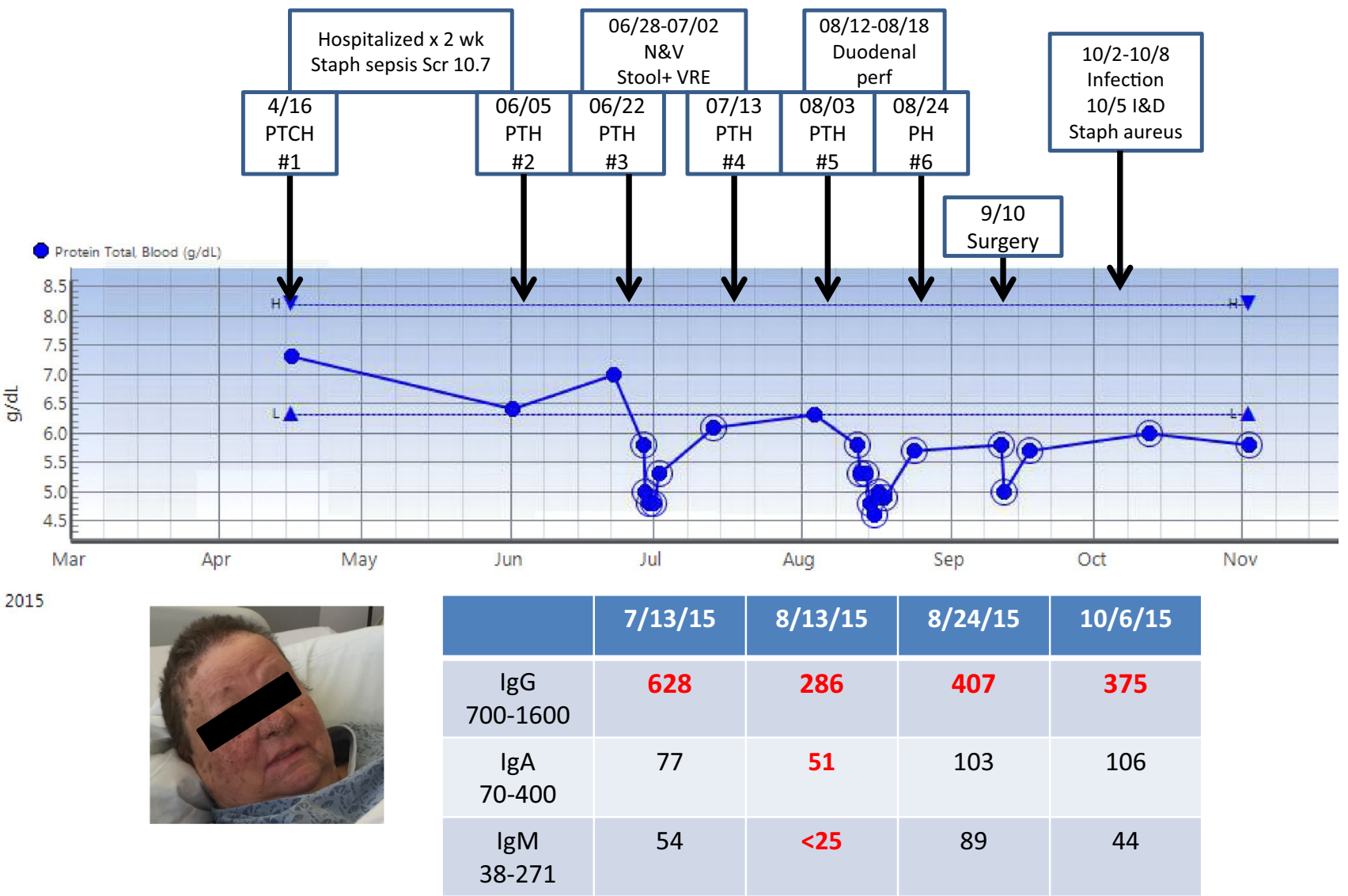

Fig. 2 A 52-year-old woman who developed sepsis following cycle 1 of chemotherapy. Treatment cycles and quantitative immunoglobulins and total protein level are charted over time

dialysis. She was discharged on day 11 with normal renal function. Subsequent chemotherapy excluded carboplatin. Following cycle 3 , she was hospitalized with nausea and vomiting, cultures were negative with the exception of vancomycin-resistant Enterococcus (VRE) in her stool that was consistent with colonization. IgG on that admission was low. Cycle 5 was complicated by a duodenal perforation but no documented infection. $\operatorname{IgG}, \operatorname{IgM}$, and $\operatorname{IgA}$ were low on that admission (what were the levels). Her final preoperative treatment included only pertuzumab and trastuzumab. The postoperative course was complicated by a wound infection requiring incision and debridement with microbiology positive for Staphylococcus aureus. Pertuzumab was deleted from her postoperative treatment and immunoglobulins returned to the normal level. She is alive and well and free of disease 39 months from diagnosis and with no further infectious or renal sequelae.

The second patient (Patient 2) presented with de novo stage IV disease treated with nab-paclitaxel, pertuzumab, and trastuzumab. Her clinical course and serum albumin levels are shown in Fig. 3. She developed an erythematous rash over her chest with the first cycle of treatment, which waxed and waned. After month 4, she was treated for a dental abscess. Over an 18-month period, she presented on three separate occasions with fever and chills and was documented to have Streptococcus agalactiae bacteremia each time and endocarditis was ruled out. The rash resolved within 2 months of stopping the pertuzumab and she has had no further infections. She is currently on maintenance trastuzumab in continued complete remission 58 months from initial diagnosis.

\section{Impact of infections on response to therapy}

Twenty-four women were treated in the neoadjuvant setting. One patient was lost to follow-up and one died prior to surgery; 23 were evaluable for treatment efficacy. The overall pathologic complete response (pCR) at definitive surgery was $68 \%$ ( 15 of 22 patients), including $10 / 16 \mathrm{HR}+$ and $5 / 6$ $\mathrm{HR}$ - patients. 


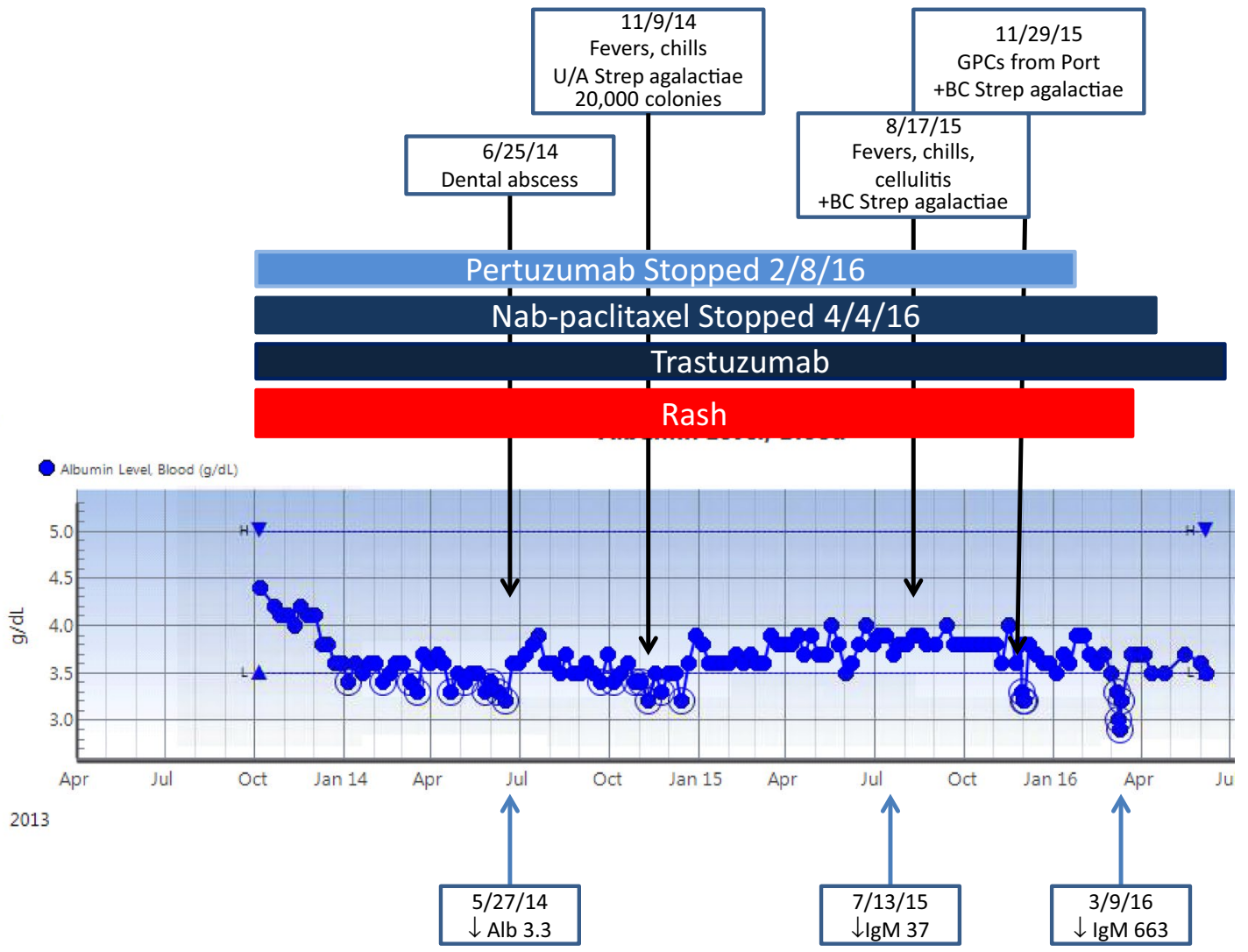

Fig. 3 A 31-year-old woman who developed a rash immediately after starting treatment and continued for over 2 years until the pertuzumab was discontinued. She developed a dental abscess and documented

\section{Discussion}

This updated series provides additional support for an association of Gram-positive bacterial infections and cutaneous manifestations with the addition of pertuzumab to trastuzumab alone or trastuzumab-based chemotherapy. Although the skin reaction is reminiscent of that observed with EGFR inhibitors, the deep-seated infections reported in this series suggest a more serious clinical problem that we believe is related to the development of hypogammaglobulinemia and perhaps abrogation of a specific immune pathway that requires further study.

Because this was a registry trial, we relied on the treating physicians to report infectious complications that were attributable to their therapy. We are not able to determine the incidence of these adverse reactions. Previously, we estimated that skin and nail infections occurred in 30\% of patients receiving pertuzumab and trastuzumab therapy and this updated case series supports that number and begs the question why this is not a recognized side effect of this therapy [1] We speculate that the skin "rash" has often been attributed to other causes such as dexamethasone or chemotherapy. Folliculitis was the most common problem
Strep Agalactiae sepsis with low albumin and low immunoglobulins which resolved with discontinuation of pertuzumab. Clinical events and immunoglobulin levels are plotted across time and albumin levels

and was often described as a "rash". Table 2 summarizes the incidence of rash reported in randomized clinical trials that tested the addition of pertuzumab to trastuzumab-based treatment, and consistently shows an increased incidence of rash in the pertuzumab arm. The CLEOPATRA trial, first reported in 2012, identified a higher incidence of rash in women assigned pertuzumab, $33.7 \%$ compared to $24.2 \%$ in those not receiving pertuzumab [2]. A more recent analysis of the CLEOPATRA included an assessment of adverse events after completion of docetaxel. The incidence of rash was higher in the pertuzumab arm, $18.3 \%$ compared to $8 \%$ [3]. Rashes are reported in a higher incidence with pertuzumab regardless of whether the comparator arm includes chemotherapy, endocrine therapy, or trastuzumab without chemotherapy.

A meta-analysis of 13 randomized trials of trastuzumab in over 10,000 women found a significantly higher risk of highgrade infections and febrile neutropenia in patients receiving trastuzumab compared to those who did not [4]. We hypothesize that the hypogammaglobulinemia (or an undefined pathway) predisposes to the development of infections with Gram-positive bacteria. In our experience, skin erythema with pustules, infection, and hypogammaglobulinemia occur 
Table 2 Incidence of rash in randomized trials in HER2 + breast cancer where the addition of pertuzumab was tested

\begin{tabular}{|c|c|c|c|c|c|}
\hline \multirow[b]{2}{*}{$\begin{array}{l}\text { Baselga, } 2012 \text { [2] } \\
\text { Med f/up } 19.3 \text { months }\end{array}$} & \multirow{2}{*}{$\begin{array}{l}\text { Population } \\
\text { First-line metastasis }\end{array}$} & \multicolumn{4}{|l|}{ Incidence of rash by treatment } \\
\hline & & Docetaxel, trastuzumab & $96 / 397(24.2 \%)$ & $\begin{array}{l}\text { Docetaxel, trastuzumab + per- } \\
\text { tuzumab }\end{array}$ & $137 / 407(33.7 \%)$ \\
\hline $\begin{array}{l}\text { Swain, } 2015 \text { [3] } \\
\text { After docetaxel dc'ed } \\
\text { med f/up } 50 \text { months }\end{array}$ & First-line metastasis & Control & $21 / 261(8 \%)$ & Trastuzumab + pertuzumab & $56 / 306(18.3 \%)$ \\
\hline Urruticoechea. 2016 [11] & $\begin{array}{l}\text { Advanced disease } \\
\text { after tras-chemo }\end{array}$ & Capecitabine + trastuzumab & $11 / 218(5 \%)$ & $\begin{array}{l}\text { Capecitabine, trastu- } \\
\text { zumab + pertuzumab }\end{array}$ & $34 / 228(14.9 \%)$ \\
\hline Rimawi, 2018 [12] & First-line metastasis & $\mathrm{AI}+$ trastuzumab & $11 / 124(8.9 \%)$ & $\begin{array}{l}\mathrm{AI}+\text { trastuzumab + pertu- } \\
\text { zumab }\end{array}$ & $22 / 127(17.3 \%)$ \\
\hline \multirow[t]{2}{*}{ Gianni, 2016 [13] } & Neoadjuvant & Docetaxel, trastuzumab & $26 / 107(24 \%)$ & $\begin{array}{l}\text { Docetaxel, trastuzumab }+ \text { per- } \\
\text { tuzumab }\end{array}$ & $30 / 107(28 \%)$ \\
\hline & & Docetaxel, pertuzumab & $30 / 94(32 \%)$ & Trastuzumab + pertuzumab & $22 / 108(20 \%)$ \\
\hline
\end{tabular}

$A I$ aromatase inhibitor

within the first 1-4 days after administration of chemotherapy and generally resolve prior to starting the next cycle of therapy. The early development of hypogammaglobulinemia could explain early onset of pustular rashes and abscesses. In the absence of serious complications, patients are not generally seen in the early post-chemotherapy period and this could also explain why others have not identified the hypogammaglobulinemia. Patient 1 was repeatedly seen shortly after chemotherapy and we were able to document low immunoglobulins on multiple occasions even without a documented infection.

Currently the FDA's "accelerated approval" process can lead to more rapid drug approval if the preliminary studies demonstrate improvement in surrogate markers of the disease, such as tumor shrinkage. Confirmatory data that address benefit to the patient are required for full FDA approval. Randomized clinical trials are designed to demonstrate the improved efficacy of a therapeutic agent and are generally not powered to identify uncommon side effects [5]. Generally toxicity is assessed during the treatment period and long-term complications are more difficult to identify. Even serious adverse events have been underestimated and many do not become apparent for decades after FDA approval $[6,7]$. It should not be surprising that adverse events, even serious adverse events, are often identified years after a drug has been on the market.

Rashes are often reported in patients treated with tyrosine kinase inhibitors (TKIs) in for a variety of cancers. Often the rash has been associated with more favorable disease outcomes and this includes lapatinib in HER2 positive breast cancer [8-10]. Our series was too small to determine whether infectious complications had any prognostic significance. Following neoadjuvant therapy, pCR at surgery was seen in $68 \%$ of patients and is comparable to the reported data.
We routinely discuss the risk of infectious complications as part of the chemotherapy teaching for women receiving regimens, which include both trastuzumab and pertuzumab. Patients who develop folliculitis are initially treated with topical clindamycin gel or oral doxycycline in more severe cases and chemotherapy can continue on schedule. It is important that infectious complications be recognized as a side effect of pertuzumab and trastuzumab, because none of our patients were neutropenic, most received white blood cell growth factors, and one of our patients died as a result of a Gram-positive bacterial infection on day 6 of her second cycle of treatment.

The data presented are from a single institution and the mechanism for this complication has yet to be elucidated. We are initiating a clinical trial to assess serial immunoglobulins and granulocyte function after treatment with regimens that include trastuzumab and pertuzumab.

Acknowledgements NCI Grant \# P30 CA33572. The authors thank Nicola Welch, PhD, CMPP for editorial assistance and critical review. Dr. Mortimer has received fees as a consultant for Puma, Eisai, and Karyopharm. Dr. Dadwal received consulting fees from Janssen, Clinigen, and Merck. He also has clinical trials funding from Merck. Dr. Jung received consulting fees from Regeron-Sanofi, Merck, and Iderra. Dr. Yuan Received consultation fees from Eisai, Genentech, AstraZeneca, and Immunomedics. She has received clinical trials funding from Eisai, Novartis, Puma, Genentech and Pfizer.

\section{Compliance ethical standards}

Conflict of interest The authors declare that they have no conflict of interest.

Ethical approval This article does not contain any studies with human participants performed by any of the authors.

Informed consent This review was approved by the Institutional Review Board and was a chart review study which did not require informed consent. 
Open Access This article is distributed under the terms of the Creative Commons Attribution 4.0 International License (http://creativeco mmons.org/licenses/by/4.0/), which permits unrestricted use, distribution, and reproduction in any medium, provided you give appropriate credit to the original author(s) and the source, provide a link to the Creative Commons license, and indicate if changes were made.

\section{References}

1. Mortimer J, Jung J, Yuan Y, Kruper L, Stewart D, Chung S, Yu K, Mendelsohn M, D'Apuzzo M, Tegtmeier B et al (2014) Skin/nail infections with the addition of pertuzumab to trastuzumab-based chemotherapy. Breast Cancer Res Treat 148(3):563-570

2. Baselga J, Bradbury I, Eidtmann H, Di Cosimo S, de Azambuja E, Aura C, Gómez H, Dinh P, Fauria K, Van Dooren V et al (2012) Lapatinib with trastuzumab for HER2-positive early breast cancer (NeoALTTO): a randomised, open-label, multicentre, phase 3 trial. The Lancet 379(9816):633-640

3. Swain SM, Baselga J, Kim S-B, Ro J, Semiglazov V, Campone M, Ciruelos E, Ferrero J-M, Schneeweiss A, Heeson S et al (2015) Pertuzumab, trastuzumab, and docetaxel in HER2-positive metastatic breast cancer. N Engl J Med 372(8):724-734

4. Funakoshi T, Suzuki M, Muss H (2015) Infection risk in breast cancer patients treated with trastuzumab: a systematic review and meta-analysis. Breast Cancer Res Treat 149(2):321-330

5. Niraula S, Amir E, Vera-Badillo F, Seruga B, Ocana A, Tannock IF (2014) Risk of incremental toxicities and associated costs of new anticancer drugs: a meta-analysis. J Clin Oncol 32(32):3634-3642

6. Ladewski LA, Belknap SM, Nebeker JR, Sartor O, Lyons EA, Kuzel TC, Tallman MS, Raisch DW, Auerbach AR, Schumock GT et al (2003) Dissemination of information on potentially fatal adverse drug reactions for cancer drugs from 2000 to 2002: first results from the research on adverse drug events and reports project. J Clin Oncol 21(20):3859-3866

7. Moore TJ, Cohen MR, Furberg CD (2007) Serious adverse drug events reported to the food and drug administration, 1998-2005. JAMA Internal Med 167(16):1752-1759
8. Zaborowska-Szmit M, Kowalski DM, Piórek A, Krzakowski M, Szmit S (2016) A decrease in D-dimer concentration and an occurrence of skin rash as iatrogenic events and complementary predictors of survival in lung cancer patients treated with EGFR tyrosine kinase inhibitors. Pharmacol Rep 68(6):1140-1148

9. Rubovszky G, Budai B, Ganofszky E, Horváth Z, Juhos É, Madaras B, Nagy T, Szabó E, Pintér T, Tóth E et al (2018) Predictive value of early skin rash in cetuximab-based therapy of advanced biliary tract cancer. Pathol Oncol Res 24(2):237-244

10. Sonnenblick A, de Azambuja E, Agbor-tarh D, Bradbury I, Campbell C, Huang Y, Dueck AC, Pritchard KI, Wolff AC, Jackisch C et al (2016) Lapatinib-related rash and breast cancer outcome in the ALTTO phase III randomized trial. JNCI J Natl Cancer Inst 108(8):1-8

11. Urruticoechea A, Rizwanullah M, Im S-A, Ruiz ACS, Láng I, Tomasello G, Douthwaite H, Crnjevic TB, Heeson S, Eng-Wong J et al (2017) Randomized phase III trial of trastuzumab plus capecitabine with or without pertuzumab in patients with human epidermal growth factor receptor 2-positive metastatic breast cancer who experienced disease progression during or after trastuzumab-based therapy. J Clin Oncol 35(26):3030-3038

12. Rimawi M, Ferrero J-M, Haba-Rodriguez JDL, Poole C, Placido SD, Osborne CK, Hegg R, Easton V, Wohlfarth C, Arpino G et al (2018) First-line trastuzumab plus an aromatase inhibitor, with or without pertuzumab, in human epidermal growth factor receptor 2-positive and hormone receptor-positive metastatic or locally advanced breast cancer (PERTAIN): a randomized, open-label phase II trial. J Clin Oncol 36(28):2826-2835

13. Gianni L, Pienkowski T, Im Y-H, Tseng L-M, Liu M-C, Lluch A, Starosławska E, de la Haba-Rodriguez J, Im S-A, Pedrini JL et al (2016) 5-year analysis of neoadjuvant pertuzumab and trastuzumab in patients with locally advanced, inflammatory, or earlystage HER2-positive breast cancer (NeoSphere): a multicentre, open-label, phase 2 randomised trial. Lancet Oncol 17(6):791-800

Publisher's Note Springer Nature remains neutral with regard to jurisdictional claims in published maps and institutional affiliations. 